\title{
Mouse Disorder by Site
}

National Cancer Institute

\section{Source}

National Cancer Institute. Mouse Disorder by Site. NCI Thesaurus. Code C26390.

An organization of mouse disorders based on the body site affected. 